\title{
Therapeutic effect of taxanes on metastatic breast cancer of various immunohistochemical subtypes
}

\author{
IPPEI FUKADA ${ }^{1}$, KAZUHIRO ARAKI ${ }^{1}$, KOKORO KOBAYASHI ${ }^{1}$, TAKAYUKI KOBAYASHI ${ }^{2}$, \\ RIE HORII $^{3}$, FUTOSHI AKIYAMA ${ }^{3}$, SHUNJI TAKAHASHI ${ }^{2}$, TAKUJI IWASE ${ }^{4}$ and YOSHINORI ITO ${ }^{1}$ \\ ${ }^{1}$ Department of Breast Medical Oncology, Breast Oncology Center,
}

The Cancer Institute Hospital of The Japanese Foundation for Cancer Research; Departments of ${ }^{2}$ Medical Oncology and

${ }^{3}$ Pathology, The Cancer Institute Hospital of The Japanese Foundation for Cancer Research; ${ }^{4}$ Breast Surgical Oncology,

Breast Oncology Center, The Cancer Institute Hospital of The Japanese Foundation for Cancer Research,

Tokyo 135-8550, Japan

Received February 3, 2015; Accepted April 29, 2016

DOI: $10.3892 / \mathrm{ol} .2016 .4627$

\begin{abstract}
Taxane drugs play a central role in chemotherapy for breast cancer. However, previous studies have reported that taxanes are relatively ineffective in patients with operable luminal breast cancer compared with other subtypes. Between January 2000 and August 2008, 293 patients with metastatic breast cancer were treated with taxanes in The Cancer Institute Hospital of The Japanese Foundation for Cancer Research and were included in the present study. The patients were divided into 4 subtypes based on the immunohistochemically evaluated estrogen receptor (ER), progesterone receptor $(\mathrm{PgR})$ and human epidermal growth factor receptor 2 (HER2) status. The clinicopathological features, response rate $(R R)$ and time to progression (TTP) were analyzed retrospectively. In total, 159 patient tissues were classified as luminal type $\left(\mathrm{ER}^{+}\right.$ and/or PgR ${ }^{+}$and HER2-), 28 patient tissues were classified as luminal-HER2 type (ER ${ }^{+}$and/or $\mathrm{PgR}^{+}$and $\left.\mathrm{HER} 2^{+}\right), 57$ patient tissues were classified as HER2 type (ER-, PgR and HER2 ${ }^{+}$), and 49 patient tissues were classified as triple-negative type (ER', PgR' and HER2-). Among the 4 subtypes, the clinical benefit rate was 51.6, 78.6, 71.9 and $40.8 \%$, respectively. There were significant differences in TTP between subtypes (median TTP, 8.3 months in luminal, 14.1 months in luminal-HER2, 10.6 months in HER2, and 4.2 months in triple-negative; $\mathrm{P}<0.001)$. Patients with luminal type tumors had a significantly longer TTP than patients with triple-negative type tumors. The present data suggested that the immunohistochemical subtypes were associated with the therapeutic effect of taxanes for metastatic breast cancer and that taxanes yielded an acceptable
\end{abstract}

Correspondence to: Dr Ippei Fukada, Department of Breast Medical Oncology, Breast Oncology Center, The Cancer Institute Hospital of The Japanese Foundation for Cancer Research, 3-8-31 Ariake, Tokyo 135-8550, Japan

E-mail: ippei.fukada@jfcr.or.jp

Key words: taxane, breast cancer, metastasis, subtype, response rate, time to progression
RR and TTP in luminal metastatic breast cancer. Additional investigations are required to elucidate the predictive markers of taxane therapy for patients with metastatic breast cancer in each immunohistochemical subtype.

\section{Introduction}

Taxane binds to $\beta$-tubulin, promotes microtubule polymerization and prevents depolymerization, thereby arresting cell division and inducing apoptosis (1). In Japan, docetaxel was approved in June 1997 and paclitaxel was approved in October 1997. Currently, taxane and anthracycline play a central role in chemotherapy for breast cancer (2).

There have been numerous studies on the therapeutic effect of taxanes in metastatic breast cancer (3,4). Jones et al (3) conducted a randomized phase III trial to compare the effects of docetaxel and paclitaxel in metastatic breast cancer, reporting that the overall response rate (ORR) and median time to progression (TTP) for docetaxel and paclitaxel were $32 \%$ and 5.7 months and $25.0 \%$ and 3.6 months, respectively (3). Gradishar et al reported that the RR and median TTP for taxane as the first-line therapy were $27 \%$ and 19.7 weeks, respectively, and that the ORR and median TTP were $19 \%$ and 16.9 weeks, respectively (4).

Gene expression profiling using DNA microarrays classifies breast cancers into five intrinsic subtypes: Luminal A, luminal B, ER BB2+, normal-like and basal-like (5). Accordingly, immunohistochemical classification with hormone receptors and human epidermal growth factor receptor 2 (HER2) may be used to estimate the subtype. Luminal A breast cancer was defined to express estrogen receptor (ER) and not express HER2 in the CALGGB 9344 trial (6), express ER and/or progesterone receptor (PgR) with a low Ki67 labeling index (cut-off, 13\%) in the BCIRG 001 trial (7), and express ER with a low Ki67 labeling index (cut-off, 20\%) in the PACS 01 trial (8). In addition, the St. Gallen International Conference 2011 proposed that breast cancer expressing ER and/or PgR, and negative for HER2 with a Ki67 labeling index of $<14 \%$, should be defined as luminal A breast cancer (9).

Numerous studies have reported that chemotherapy is relatively ineffective in patients with early luminal A breast 
cancer. Pritchard et al reported that treatment containing anthracycline was not superior to cyclophosphamide, methotrexate and 5-fluorouracil in HER2- breast cancer (10). The BCIRG 001 trial showed that docetaxel, doxorubicin and cyclophosphamide (TAC) chemotherapy was more effective compared with fluorouracil, doxorubicin and cyclophosphamide (FAC) chemotherapy, but failed to show superiority in patients with luminal A breast cancer (7). Meta analysis of the EBCTCG trials showed that the proportional risk reductions of chemotherapy were observed equally in patients with ER+ and ER- breast cancer in a group of women aged $<50$ years (11). However, the NSABP B-30 trial followed up the premenopausal women of patients who had received chemotherapy, and found that the hazards ratio for disease-free survival was reduced subsequent to menopause in premenopausal patients that had received chemotherapy. This suggested that the chemotherapy-induced depletion of endogenous estrogen may be more important than the effect of chemotherapy itself (12). Alba et al directly compared the efficacy of aromatase inhibitors and chemotherapy with epirubicin and cyclophosphamide followed by taxane in neoadjuvant therapy for $\mathrm{ER}$ or $\mathrm{PgR}^{+}$and HER2- luminal breast cancer, finding that the RR and complete histological RR of chemotherapy were not significantly higher than those for endocrine therapy (13).

In addition, several studies have reported the absence of an additional benefit of taxane treatment for luminal breast cancer. The CALGB 9344 trial showed that in luminal A breast cancer, defined as breast cancer positive for ER and negative for HER2, there was no additional benefit of the addition of paclitaxel to doxorubicin and cyclophosphamide chemotherapy (6). The BCIRG 001 trial (7), in which luminal A breast cancer was defined as breast cancer positive for ER or PgR, with a low Ki67 labeling index (cut-off, 13\%), reported that the prognosis of patients treated with TAC was approximately equal to that of patients treated with FAC. The PACS 01 trial (8) compared 6 cycles of 5-flourouracil, epirubicin and cyclophosphamide (FEC) and 3 cycles of FEC followed by 3 cycles of docetaxel. This study found that the recurrence-free survival of FEC followed by docetaxel was not superior to FEC alone in patients with luminal A breast cancer (defined as breast cancer positive for ER with a Ki67 labeling index <20\%).

Overall, numerous studies have suggested that taxane therapy is relatively ineffective in patients with operable early luminal breast cancer. However, to the best of our knowledge, no studies have reported whether taxane also lacks efficacy as a treatment for luminal metastatic breast cancer. Therefore, the therapeutic effect of taxanes on metastatic breast cancer in various immunohistochemical subtypes was retrospectively investigated.

\section{Materials and methods}

Patient selection. Of the 527 patients with metastatic breast cancer treated in The Cancer Institute Hospital of The Japanese Foundation for Cancer Research (Tokyo, Japan) between January 2000 and August 2008, 293 patients received treatment with taxanes. Out of these patients, 159 patient tissues were classified as luminal type (ER ${ }^{+}$and/or $\mathrm{PgR}^{+}$and HER2), 28 patient tissues were classified as luminal HER2 type $\left(\mathrm{ER}^{+}\right.$ and/or $\mathrm{PgR}^{+}$and $\mathrm{HER}_{2}^{+}$), 57 patient tissues were classified as
HER 2 type $\left(\mathrm{ER}^{-}, \mathrm{PgR}^{-}\right.$and $\left.\mathrm{HER} 2^{+}\right)$, and 49 patient tissues were classified as triple-negative type (ER $\mathrm{PgR}^{-}$and $\left.\mathrm{HER} 2^{-}\right)$breast cancer.

Methods. Comprehensive consent for the use of specimen materials was obtained preoperatively from all patients participating as subjects in the present study. The study was approved by the Institutional Review Board of The Cancer Institute Hospital of The Japanese Foundation for Cancer Research (Tokyo, Japan).

The immunohistochemical subtypes were determined by biopsy of the primary lesion at stage IV and the surgical materials in recurrent breast cancer. Biopsy specimens were embedded in paraffin and cut into $4-\mu \mathrm{m}$ slices. The specimens were stained with hematoxylin and eosin (HE) and were immunohistochemically examined for ER, PgR and HER2 expression. Using HE-stained slices, the histological subtypes of cancer and the nuclear grade of cancer cells were evaluated according to the general rules for clinical and pathological assessment of breast cancer, as edited by the Japanese Breast Cancer Society (14). The histological subtype was divided into three groups, consisting of papillotubular carcinoma, solid-tubular carcinoma and scirrhous carcinoma, based on the size and structure of invasive components. The definitions of the histological subtypes were as follows: Papillotubular carcinoma, an invasive carcinoma characterized by large invasive components with papillary structures and/or tubular formations; solid-tubular carcinoma, an invasive carcinoma characterized by large solid invasive components; and scirrhous carcinoma, an invasive carcinoma characterized by small scattered invasive components or trabecular invasive nests with a desmoplastic stroma. The nuclear grade was evaluated using a combination of nuclear atypia and mitotic counts. Nuclear grade was divided into three groups, consisting of nuclear grades 1, 2 and 3 , in order of increasing atypia.

Immunohistochemical assessment of ER and PgR expression was performed using mouse anti ER $\alpha$ monoclonal antibody (product no. IR08461, clone, 1D5; Dako Japan Co., Ltd., Tokyo, Japan. This product is supplied in $0.05 \mathrm{~mol} / \mathrm{l}$ Tris/HCl, pH 7.2, containing $15 \mathrm{~mol} / 1$ sodium azide and fetal calf serum protein (1 ml total volume). Anti-ER, 1D5 used at a dilution of 1:35 when performing IHC using the Dako EnVision) and mouse anti PgR monoclonal antibody (product no. IS06830, clone, PgR636; Dako Japan Co., Ltd. Ready-to-use monoclonal mouse antibody provided in liquid form in a buffer containing stabilizing protein and $0.015 \mathrm{~mol} / \mathrm{l}$ $\mathrm{NaN}_{3}$. The target concentration of Anti-PR, clone PgR636 is $0.5 \mu \mathrm{g} / \mathrm{ml}$; the acceptable concentration range of Anti-PR, clone PgR636 is $0.4-0.6 \mu \mathrm{g} / \mathrm{ml})$. Positive reactions for ER and $\mathrm{PgR}$ were defined as nuclear staining in $\geq 10 \%$ of cancer cells, and no reaction was defined as staining in $<10 \%$ of cancer cells. Hormone receptor positivity was defined as showing positivity for ER or PgR. Immunohistochemical detection of the HER2 protein was performed using the HercepTest (product no. K520411, Dako Japan Co., Ltd.).

Immunohistochemical detection of the HER2 protein was performed using the HercepTest (Dako Japan Co., Ltd.). Expression of the HER2 protein was classified into four groups: $0 ; 1+; 2+$; and $3+$. In the tissues that were classified as 
Table I. Patient characteristics $(n=293)$.

\begin{tabular}{lc}
\hline Patient characteristics & Value, $\mathrm{n}(\%)$ \\
\hline Age, years & \\
Median & 54 \\
Range & $28-84$
\end{tabular}

Age distribution

$<50$

$112(38.2)$

$\geq 50$

$181(61.8)$

Status

Stage IV

$92(31.4)$

Recurrence

201 (68.6)

DFI, days

1,323

Number of metastasis

Median

Range

$0-8$

Sites of metastasis

Lymph node

$190(64.8)$

Liver

$138(47.1)$

Lung

$156(53.2)$

Bone

$178(60.8)$

Brain

$69(23.5)$

$64(21.8)$

Local

$51(17.4)$

Prior chemotherapy regimens

None

65 (22.2)

1

2

3

$\geq 4$

103 (35.2)

70 (23.9)

32 (10.9)

$23(7.8)$

Prior anthracycline

143 (48.8)

For metastasis

NAC or adjuvant

135 (46.1)

8 (2.7)

Estrogen receptor

Positive

$177(60.4)$

Negative

$116(39.6)$

Progesterone receptor

Positive

133 (45.4)

Negative

160 (54.6)

HER2 status

IHC 0

114 (38.9)

IHC $1+$

77 (26.3)

IHC 2+

FISH negative

FISH positive

$32(10.9)$

$18(6.1)$

$14(4.8)$

IHC $3+$
2+, HER2 genetic testing by fluorescent in situ hybridization (FISH) was performed using a PathVysion HER2-DNA Probe kit (Abbott Molecular, Inc., Des Plaines, IL, USA). The protein and genetic status were each estimated based on the guidelines for HER2 testing in breast cancer, as edited by the American Society of Clinical Oncology/College of American Pathologists (15). HER2 positivity was defined as HER2 protein expression of $3+$ or HER 2 gene amplification.

Based on the combination of ER, PgR and HER2 expression, patients were classified into four subtypes, defined as follows: Luminal subtype, $\mathrm{ER}^{+}$and/or $\mathrm{PgR}^{+}$, and HER2; luminal HER2 subtype, $\mathrm{ER}^{+}$and/or $\mathrm{PgR}^{+}$, and HER2 ${ }^{+}$; HER2 type, $\mathrm{ER}^{-}, \mathrm{PgR}^{-}$and $\mathrm{HER}^{+}$; and triple-negative subtype, ER ${ }^{-}$, PgR' and HER2-.

Treatment. The taxane regimens employed in the present study included a weekly paclitaxel (wPAC) regimen at a dose of $80 \mathrm{mg} / \mathrm{m}^{2}$ and a triweekly docetaxel (3wDOC) regimen at a dose of $60 \mathrm{mg} / \mathrm{m}^{2}$, which is the approved dose of docetaxel in Japan. wPAC and 3wDOC were administered to 59.1 and $40.9 \%$ of the patients with luminal type breast cancer, 78.6 and $21.4 \%$ of the patients with luminal-HER 2 type breast cancer, 82.5 and $17.5 \%$ of the patients with HER 2 type breast cancer, and 55.1 and $44.9 \%$ of the patients with triple-negative type breast cancer, respectively. Concurrent trastuzumab therapy was administered to $89.3 \%$ of the patients with luminal-HER2 type breast cancer, $94.7 \%$ of the patients with HER2 type breast cancer and $1.9 \%$ of the patients with luminal type breast cancer who were later found to not express HER2 by FISH. In patients who received wPAC and 3wDOC for the treatment of metastatic breast cancer, the therapeutic effect of the initial regimen including a taxane was evaluated.

Response evaluation. Radiological tumor assessments were performed by computed tomography every 3-4 months during treatment. Local lesion and lymph node metastases were measured by echogram. The response to chemotherapy was assessed according to the Response Evaluation Criteria in Solid Tumors (RECIST) version 1.1 (16). TTP was defined as the time interval between the start of taxane treatment and disease progression or failure of treatment due to adverse events.

Statistical analysis. SPSS version 17.0 (SPSS, Inc., Chicago, IL, USA) was used for statistical analysis in the present study. The ORR and clinical benefit rate (CBR) were analyzed using the $\chi^{2}$ test. TTP was tested by the log-rank test using the Kaplan-Meier method. $\mathrm{P}<0.05$ was considered to indicate a statistically significant difference.

\section{Results}

Patient characteristics. The characteristics of all 293 patients are listed in Table I. All evaluated patients were diagnosed with invasive breast cancer. The average age was 54 years. The patient numbers according to the age group were as follows: $\leq 49$ years old, 112 patients $(38.2 \%)$; and $\geq 50$ years old, 181 patients $(61.8 \%)$. The most common sites of distant metastasis were the lymph nodes, which was identified in 190 patients $(64.8 \%)$. The sites of metastases was 3

DFI, disease free interval; NAC, neoadjuvant chemotherapy; HER2, human epidermal growth factor receptor type 2; IHC, Immunohistochemistry; FISH, fluorescence in situ hybridization. 


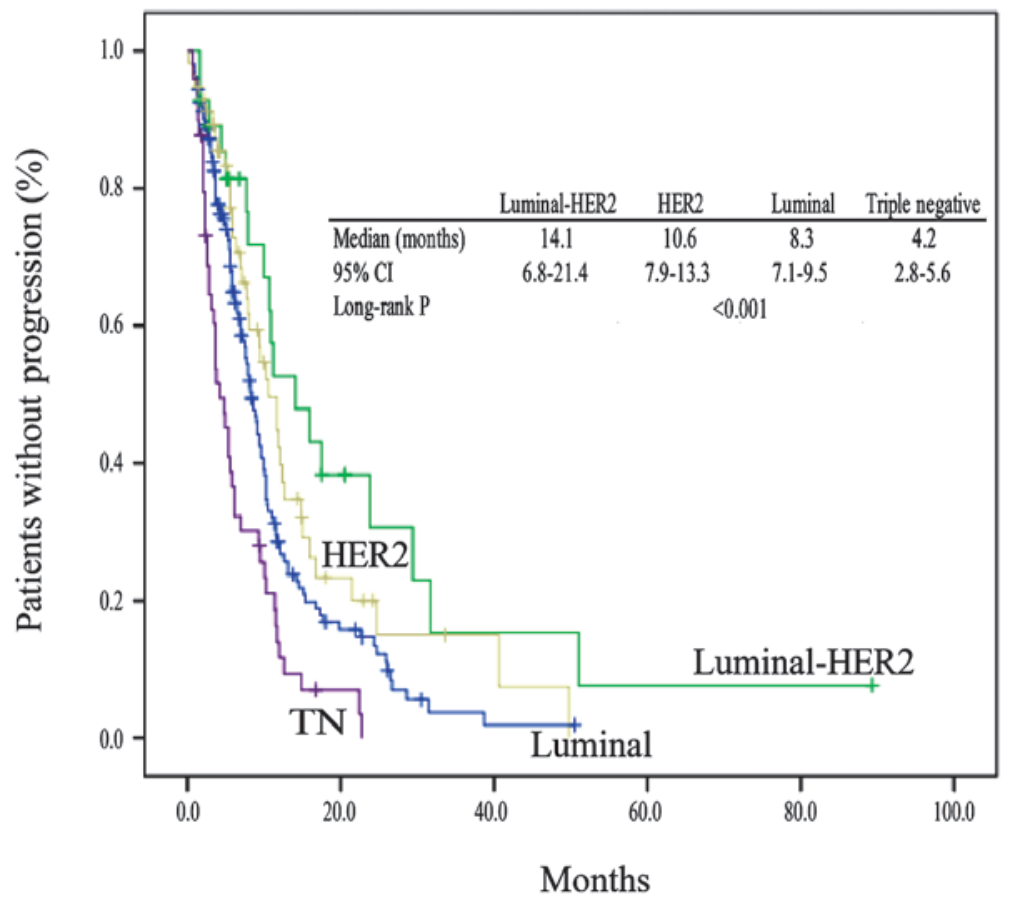

Figure 1. TTP in the 4 breast cancer subtypes. There were significant differences between the TTP in the 4 subtypes $(\mathrm{P}<0.001)$. Among the four subtypes, patients with the luminal-HER2 type had a significantly improved TTP (median TTP, 14.1 months). The TTPs of patients with luminal and HER2 tumors (8.3 and 10.6 months) were compared with the TTP of patients with triple-negative tumors (4.2 months). TTP, time to progression; HER2, human epidermal growth factor receptor type 2; TN, triple-negative.

$(\mathrm{P}=0.016)$. The number of patients that received one previous chemotherapy regimen was 103 patients $(35.2 \%$; $\mathrm{P}=0.003)$; 143 patients $(48.8 \%)$ had a history of receiving anthracyclines $(\mathrm{P}<0.001)$.

Distribution of immunohistochemical subtypes and clinicopathological features. Associations between the distribution of immunohistochemical subtypes and clinicopathological features are shown in Table II. While there was no significant difference in age among the 4 subtypes, significant differences between subtype distribution, number of metastases, number of prior chemotherapy regimens and history of anthracycline administration were observed. Luminal type tumors were associated with a significantly increased incidence of bone metastasis $(\mathrm{P}<0.001)$, while the HER2 and triple-negative types showed a significantly increased frequency of brain metastasis (both $\mathrm{P}<0.001)$. The patients with luminal type tumors had received more prior chemotherapy compared with other subtypes, and the luminal type included a significantly increased proportion of patients with a history of receiving anthracyclines $(\mathrm{P}<0.001)$.

$R R$. The RR and CBR in the 4 subtypes are shown in Table III. The RR was $33.1 \%$ and the CBR was $56.3 \%$ in all patients with metastatic breast cancer. The RR and CBR in the 4 subtypes were significantly different between the subtypes $(\mathrm{P}<0.001)$. In the luminal-HER2 and HER2 types, the RRs were 57.1 and $52.6 \%$, respectively, and the CBRs were 78.6 and $71.9 \%$, respectively. In the luminal type, the RR and CBR were 28.3 and $51.6 \%$, respectively. Between the luminal and triple-negative types, the RR and CBR of patients with luminal type tumors were increased compared with the patients with triple-negative type tumors.
TTP according to subtypes. The median follow-up time was 9.2 months. TTP events had occurred in 117 patients with luminal type tumors (73.6\%), 19 patients with luminal-HER2 type tumors $(67.9 \%), 38$ patients with HER2 type tumors $(66.7 \%)$ and 45 patients with triple-negative type tumors (91.8\%). The TTP in the 4 subtypes is shown in Fig. 1. There were significant differences in TTP between subtypes (median TTP, 8.3 months in the luminal, 14.1 months in the luminal-HER2, 10.6 months in the HER2, and 4.2 months in the triple-negative types; $\mathrm{P}<0.001)$. Among the four subtypes, patients with the luminal-HER2 type had a significantly improved TTP. The TTP of patients with luminal-HER2 tumors was better than the TTP of patients with triple-negative tumors.

Adverse events leading to the discontinuation of taxanes. In $30(10.2 \%)$ of the 293 patients, taxane administration was discontinued due to adverse events (Grade 3 and 4; Table IV). PAC was discontinued due to events including peripheral neuropathy, which was most frequent at $6.3 \%$, worsening of the general condition, neutropenia and interstitial pneumonia. DOC was discontinued due to edema, which was most frequent at $6.8 \%$, nasolacrimal duct stenosis, muscle pain and heart failure.

\section{Discussion}

In the present study, the therapeutic effect of taxanes on metastatic breast cancer was retrospectively investigated in various immunohistochemical subtypes. It was found that the immunohistochemical subtypes were associated with the therapeutic effect of taxanes for metastatic breast cancer. In 
Table II. Immunohistochemical subtypes distribution.

\begin{tabular}{|c|c|c|c|c|c|}
\hline Characteristic & Luminal, n (\%) & Luminal-HER2, n (\%) & HER2, n (\%) & Triple-negative, n (\%) & P-value \\
\hline Total & $159(100.0)$ & $28(100.0)$ & $57(100.0)$ & $49(100.0)$ & \\
\hline \multicolumn{6}{|l|}{ Age, years } \\
\hline Median & 53 & 51 & 55 & 55 & \\
\hline Range & $28-84$ & $33-83$ & $34-74$ & $29-84$ & \\
\hline Age distribution & & & & & 0.155 \\
\hline$<50$ years & $70(44.0)$ & $10(35.7)$ & $17(29.8)$ & $15(30.6)$ & \\
\hline$\geq 50$ years & $89(56.0)$ & $18(64.3)$ & $40(70.2)$ & $34(69.4)$ & \\
\hline Status & & & & & 0.133 \\
\hline Stage IV & $42(26.4)$ & $13(46.4)$ & $21(36.8)$ & $16(32.7)$ & \\
\hline Recurrence & 117 (73.6) & $15(53.6)$ & $36(63.2)$ & $33(67.3)$ & \\
\hline DFI, days & 1,766 & 1,293 & 769 & 694 & \\
\hline \multicolumn{6}{|l|}{ No. of metastases, $n$} \\
\hline Median & 2.3 & 2.8 & 3.1 & 4.0 & \\
\hline Range & $1-6$ & $1-8$ & $1-7$ & $1-7$ & \\
\hline Site of metastasis & & & & & 0.016 \\
\hline Lymph node & $96(60.4)$ & $18(64.3)$ & $40(70.2)$ & $36(73.5)$ & \\
\hline Liver & $80(50.3)$ & $12(42.9)$ & $25(43.9)$ & $21(42.9)$ & \\
\hline Lung & $80(50.3)$ & $11(39.3)$ & $33(57.9)$ & $32(65.3)$ & \\
\hline Bone & 114 (71.7) & $15(53.6)$ & $24(42.1)$ & $25(51.0)$ & \\
\hline Brain & $22(13.8)$ & $5(17.9)$ & $22(38.6)$ & $20(40.8)$ & \\
\hline Local & $28(17.6)$ & $4(14.3)$ & $14(24.6)$ & $18(36.7)$ & \\
\hline Other & $26(16.4)$ & $5(17.9)$ & $8(14.0)$ & $12(24.5)$ & \\
\hline $\begin{array}{l}\text { No. of chemotherapy } \\
\text { regimens }\end{array}$ & & & & & 0.003 \\
\hline None & $21(13.2)$ & $8(28.6)$ & $20(35.1)$ & $16(32.7)$ & \\
\hline 1 & $55(34.6)$ & $9(32.1)$ & $19(33.3)$ & $20(40.8)$ & \\
\hline 2 & $39(24.5)$ & $9(32.1)$ & $12(21.1)$ & $10(21.4)$ & \\
\hline 3 & $26(16.4)$ & $1(3.6)$ & $4(7.0)$ & $1(2.0)$ & \\
\hline$\geq 4$ & $18(11.3)$ & $1(3.6)$ & $2(3.5)$ & $2(4.1)$ & \\
\hline $\begin{array}{l}\text { Prior anthracycline } \\
\text { administration }\end{array}$ & & & & & $<0.001$ \\
\hline For metastasis & $90(56.6)$ & $8(28.6)$ & $24(42.1)$ & $17(34.7)$ & \\
\hline NAC or adjuvant & $34(21.4)$ & $1(3.6)$ & $0(0.0)$ & $3(6.1)$ & \\
\hline \multicolumn{6}{|l|}{ Chemotherapy regimen } \\
\hline Weekly Paclitaxel & $94(59.1)$ & $22(78.6)$ & $47(82.5)$ & $27(55.1)$ & \\
\hline Triweekly Docetaxel & $65(40.9)$ & $6(21.4)$ & $10(17.5)$ & $22(44.9)$ & \\
\hline With Trastuzumab & $3(1.9)$ & $25(89.3)$ & $54(94.7)$ & $0(0.0)$ & \\
\hline
\end{tabular}

DFI, disease free interval (median); NAC, neoadjuvant chemotherapy; HER2, human epidermal growth factor receptor type 2.

addition, , taxane yielded the therapeutic effect of RR; $28.3 \%$ and TTP; 8.3 months in luminal metastatic breast cancer. The results of the present analysis were not comparable to the numerous previous studies (6-8), which showed the ineffectiveness of taxane anticancer drugs for operable early luminal breast cancer.

Possible causes of the difference in the results of pre- and postoperative taxane treatment for early breast cancer and those of taxane treatment for luminal metastatic breast cancer include the following factors. The first is the increased heterogeneity of luminal-type cancer cells. $\mathrm{ER}^{+}$and HER2- luminal breast cancer grows slowly, showing a low apoptosis rate and low genetic instability (17). Foulkes et al (17) proposed the hypothesis that slow-growing tumors cause heterogeneity within the tumor, such that numerous tumor cells acquire the ability for distant metastasis. Therefore, in recurrent disease, luminal breast cancer cells would be expected to be more aggressive and proliferative, and then sensitive to chemotherapy.

The second possibly cause is the heterogeneity of breast cancer biopsy specimens, which may cause 
Table III. Response rate.

\begin{tabular}{lccccr}
\hline Response & Luminal, $\mathrm{n}$ & Luminal-HER2, $\mathrm{n}$ & HER2, $\mathrm{n}$ & Triple-negative, $\mathrm{n}$ & Total, $\mathrm{n}$ \\
\hline CR & 2 & 0 & 3 & 0 & 5 \\
PR & 43 & 16 & 27 & 6 & 92 \\
SD & 78 & 8 & 18 & 25 & 129 \\
Long SD & 37 & 6 & 11 & 14 & 68 \\
PD & 36 & 4 & 9 & 18 & 67 \\
RR, $\%$ & 28.3 & 57.1 & 52.6 & 12.2 & 33.1 \\
CBR, $\%$ & 51.6 & 78.6 & 71.9 & 40.8 & 56.3 \\
\hline
\end{tabular}

$\mathrm{CR}$, complete response; PR, partial response; $\mathrm{SD}$, stable disease; PD, progressive disease; RR, response rate; CBR, clinical benefit rate; HER2, human epidermal growth factor receptor type 2. Long SD is defined as continuous SD for $>6$ months.

Table IV. Adverse events leading to the discontinuation of taxanes.

\begin{tabular}{lcc}
\hline Adverse event & Paclitaxel, $\mathrm{n}(\%)$ & Docetaxel, $\mathrm{n}(\%)$ \\
\hline Sensory neuropathy & $12(6.3)$ & $1(1.0)$ \\
Nail pain & $1(0.5)$ & $0(0.0)$ \\
Thrombocythemia & $1(0.5)$ & $0(0.0)$ \\
Neutropenia & $1(0.5)$ & $0(0.0)$ \\
Fatigue & $1(0.5)$ & $0(0.0)$ \\
Fever & $1(0.5)$ & $0(0.0)$ \\
Intestinal pneumonia & $1(0.5)$ & $0(0.0)$ \\
Edema & $1(0.5)$ & $7(6.8)$ \\
Obstruction of & $0(0.0)$ & $1(1.0)$ \\
nasolacrimal canal & & $1(1.0)$ \\
Muscular pain & $0(0.0)$ & $1(1.0)$ \\
Heart failure & $0(0.0)$ & $13(12.6)$ \\
Total & $19(10.0)$ & \\
\hline
\end{tabular}

phenotypic changes associated with metastasis or recurrence. Commonly, in stage IV disease, a biopsy of the primary lesion is performed in only a small portion of the primary cancer. By contrast, in patients with recurrent disease, the whole primary lesion is evaluated for the cancer subtype, but different subtypes may occur at the sites of metastasis and recurrence. Amir et al (18) reported that the phenotypes of hormone receptors and HER2 were different between the primary and metastatic or recurrent lesions, and that ER, PgR and HER 2 phenotypes were different between the primary and metastatic lesions in 16, 30 and $13 \%$ of patients, respectively (18). Since biopsies were not generally performed during treatment in the present study, the status of immunohistochemical expression of hormone receptors and HER2 at the site of metastasis was confirmed in only 9 patients with luminal breast cancer. The status of ER expression was identical in all primary and metastatic lesions, but the status of $\mathrm{PgR}$ expression was different between the primary and metastatic lesions in 6 patients. The HER 2 expression status in the metastatic lesions in 2 patients was $2+$ according to immunohistochemical analysis, but FISH analysis showed that the expression of HER2 was amplified and absent in
1 lesion each. Although no association between discordance of phenotypes and therapeutic effect has been elucidated, it may be important in the future to perform numerous tissue biopsies and re-evaluate the phenotypes of hormone receptor and HER 2 expression. The phenotype of metastatic lesions may alter during cancer progression. Future investigation is required to address whether information about metastatic sites obtained from biopsies may aid in the prediction of response or outcome.

In the present study, 89.3 and $94.7 \%$ of luminal-HER2 and HER2 type breast cancer patients, respectively, received a treatment regimen including trastuzumab with taxanes. Adding trastuzumab to taxane therapy would be expected to improve the TTP in these subtypes. Marty et al (19) reported the efficacy of trastuzumab combined with docetaxel as the first-line treatment in HER2 ${ }^{+}$metastatic breast cancer (19). Trastuzumab plus docetaxel was significantly superior to docetaxel alone in terms of overall RR (61 vs. 34\%) and time to disease progression (median, 11.7 vs. 6.1 months). Although the proportion of patients administered with taxanes and trastuzumab as the first-line treatment was 28.6 and $35.1 \%$ in the luminal HER2 and HER2 types, respectively, the results of the present analysis were comparable to previous studies (19). The TTP of the patients with triple-negative type breast cancer was significantly shorter compared with the TTP of patients with the other three subtypes of breast cancer. The poor prognosis of triple-negative breast cancer may be affected by the short disease-free interval.

The limitation of the present study is that there is no information on the proliferative marker index, such as Ki-67, of the primary sites. The patients with luminal breast cancer that were examined in the present study harbored the two subtypes of low-proliferative luminal A and high-proliferative luminal B. The proliferative index of primary sites may aid the identification of the response to taxanes. However, in recurrent breast cancer, breast cancer diagnosed as luminal $\mathrm{A}$ at the time of surgery was treated with endocrine therapy (20). Even for luminal B breast cancer, if not life-threatening, endocrine therapy is chosen along the algorithm of Hortobagyi (20). The present study showed that the inactivity of taxane in hormone-receptor positive or HER2 breast cancer could not be predicted at the primary site. Therefore, metastatic hormone receptor-positive breast cancer was defined as luminal breast 
cancer without the strict distinction between luminal $\mathrm{A}$ and luminal B at the time of surgery.

In taxane therapy, adverse events may cause dose reduction or discontinuation of treatment. The main adverse events that disturb treatment include paclitaxel-associated peripheral neuropathy or docetaxel-associated generalized edema. In the present study, taxane administration was discontinued due to adverse events in 30 (10.2\%) of the 293 patients. Therefore, the effect of toxicities on the efficacy of treatment with taxane in the present study may be minimal. This observation is clinically important because the present study showed that taxane remains a reasonable choice for the treatment of luminal metastatic breast cancer. Additional investigations are required to elucidate the predictive markers of taxane therapy for the patients with metastatic breast cancer in each immunohistochemical subtype.

\section{References}

1. De Weger VA, Beijnen JH and Schellens JH: Cellular and clinical pharmacology of the taxanes docetaxel and paclitaxel - A review. Anticancer Drugs 5: 488-494, 2014.

2. Gradishar WJ, Anderson BO, Balassanian R, Blair SL, Burstein HJ, Cyr A, Elias AD, Farrar WB, Forero A, Giordano SH, et al: Invasive Breast Cancer Version 1.2016, NCCN Clinical Practice Guidelines in Oncology. J Natl Compr Canc Netw 14: 324-354, 2016

3. Jones SE, Erban J, Overmoyer B, Budd GT, Hutchins L, Lower E, Laufman L, Sundaram S, Urba WJ, Pritchard KI, et al: Randomized phase III study of docetaxel compared with paclitaxel in metastatic breast cancer. J Clin Oncol 23: 5542-5551, 2005.

4. Gradishar WJ, Tjulandin S, Davidson N, Shaw H, Desai N, Bhar P, Hawkins M and O'Shaughnessy J: Phase III trial of nanoparticle albumin-bound paclitaxel compared with polyethylated castor oil-based paclitaxel in women with breast cancer. J Clin Oncol 23: 7794-7803, 2005.

5. Perou CM, Sørlie T, Eisen MB, van de Rijn M, Jeffrey SS, Rees CA, Pollack JR, Ross DT, Johnsen H, Akslen LA, et al: Molecular portraits of human breast tumors. Nature 406: 747-752, 2000.

6. Hayes DF, Thor AD, Dressler LG, Weaver D, Edgerton S, Cowan D, Broadwater G, Goldstein LJ, Martino S, Ingle JN, et al; Cancer and Leukemia Group B (CALGB) Investigators: HER2 and response to paclitaxel in node-positive breast cancer. N Engl J Med 357: 1496-1506, 2007.

7. Hugh J, Hanson J, Cheang MC, Nielsen TO, Perou CM, Dumontet C, Reed J, Krajewska M, Treilleux I, Rupin M, et al: Breast cancer subtypes and response to docetaxel in node-positive breast cancer: Use of an immunohistochemical definition in BCIRG 001 trial. J Clin Oncol 27: 1168-1176, 2009.

8. Penault-Llorca F, André F, Sagan C, Lacroix-Triki M, Denoux Y, Verriele V, Jacquemier J, Baranzelli MC, Bibeau F, Antoine M, et al: Ki67 expression and docetaxel efficacy in patients with estrogen receptor-positive breast cancer. J Clin Oncol 27: 2809-2815, 2009.
9. Goldhirsch A, Wood WC, Coates AS, Gelber RD, Thürlimann B and Senn HJ; Panel members: Strategies for subtypes - dealing with the diversity of breast cancer: Highlights of the St. Gallen International Expert Consensus on the Primary Therapy of Early Breast Cancer 2011. Ann Oncol 22: 1736-1747, 2011.

10. Pritchard KI, Shepherd LE, O'Malley FP, Andrulis IL, Tu D, Bramwell VH and Levine MN; National Cancer Institute of Canada Clinical Trials Group: HER2 and responsiveness of breast cancer to adjuvant chemotherapy. N Engl J Med 354: 2103-2111, 2006

11. Early Breast Cancer Trialists' Collaborative Group (EBCTCG): Effects of chemotherapy and hormonal therapy for early breast cancer on recurrence and 15-year survival: An overview of the randomized trials. Lancet 365: 1687-1717, 2005.

12. Swain SM, Jeong JH, Geyer CE Jr, Costantino JP, Pajon ER, Fehrenbacher L, Atkins JN, Polikoff J, Vogel VG, Erban JK, et al: Longer therapy, iatrogenic amenorrhea and survival in early breast cancer. N Engl J Med 362: 2053-2065, 2010.

13. Alba E, Calvo L, Albanell J, De la Haba JR, Arcusa Lanza A, Chacon JI,Sanchez-Rovira P,Plazaola A,Lopez Garcia-Asenjo JA, Bermejo B, et al; GEICAM: Chemotherapy (CT) and hormonotherapy (HT) as neoadjuvant treatment in luminal breast cancer patients: Results from the GEICAM/2006-03, a multicenter, randomized, phase-II study. Ann Oncol 23: 3069-3074, 2012.

14. Sakamoto G, Inaji H, Akiyama F, Haga S, Hiraoka M, Inai K, Iwase T, Kobayashi S, Sakamoto G, Sano M, et al; Japanese Breast Cancer Society: General rules for clinical and pathological recording of breast cancer 2005. Breast Cancer 12 (Suppl): S1-S27, 2005.

15. Wolff AC, Hammond ME, Hicks DG, Dowsett M, McShane LM, Allison KH, Allred DC, Bartlett JM, Bilous M, Fitzgibbons P, et al; American Society of Clinical Oncology; College of American Pathologists: Recommendations for human epidermal growth factor receptor 2 testing in breast cancer: American Society of Clinical Oncology/College of American Pathologists clinical practice guideline update. Arch Pathol Lab Med 138: 241-256, 2014.

16. Eisenhauer EA, Therasse P, Bogaerts J, Schwartz LH, Sargent D, Ford R, Dancey J, Arbuck S, Gwyther S, Mooney M, et al: New response evaluation criteria in solid tumours: revised RECIST guideline (version 1.1). Eur J Cancer 45: 228-247, 2009.

17. Foulkes WD, Reis-Filho JS and Narod SA: Tumor size and survival in breast cancer - a reappraisal. Nat Rev Clin Oncol 7: 348-353, 2010.

18. Amir E, Miller N, Geddie W, Freedman O, Kassam F, Simmons C, Oldfield M, Dranitsaris G, Tomlinson G, Laupacis A, et al: Prospective study evaluating the impact of tissue confirmation of metastatic disease in patients with breast cancer. J Clin Oncol 30: 587-592, 2012

19. Marty M, Cognetti F, Maraninchi D, Snyder R, Mauriac L, Tubiana-Hulin M, Chan S, Grimes D, Antón A, Lluch A, et al: Randomized phase II trial of the efficacy and safety of trastuzumab combined with docetaxel in patients with human epidermal growth factor receptor 2-positive metastatic breast cancer administered as first-line treatment: The M77001 study group. J Clin Oncol 23: 4265-4274, 2005.

20. Gabriel N. Hortobagyi. Drug therapy: Treatment of breast cancer. N Engl J Med 339: 974-984, 1998. 